\title{
O uso das resinas compostas tipo bulk fill: Uma revisão de literatura
}

\author{
The use of bulk fill type compound resins: A literature review
}

El uso de resinas compuestas de tipo bulk fill: Uma revisión de la literatura

José Milton de Aquino e Silva Neto ${ }^{1 *}$, Lucas Rodrigues Santana ${ }^{1}$, Kyvia Fernanda Ferreira Lima ${ }^{1}$, Michelle Leão Bittencourt Brandão Medeiros ${ }^{1}$, Tayguara Cerqueira Cavalcanti ${ }^{1}$.

\section{RESUMO}

Objetivo: Avaliar a utilização das Resinas Compostas do tipo bulk fill (RCBF) demonstrando suas indicações e ressaltar a técnica de seus procedimentos no que se refere a resistências e contrações na fotoativação. Métodos: Tratou-se de uma revisão de literatura, com abordagem descritiva com caráter informativo, onde o processo de formulação se deu através de buscas por literaturas cientificas, em bases de dados, tendo como descritores: A importância da resina composta, a forma de polimerização, potência de irradiação e dureza. Resultados: Quando contraposta às resinas compostas convencionais, frequentemente apontadas para adição em incrementos de $2 \mathrm{~mm}$, no extremo, as RCBF expressaram padrões de vigor melhor. Porém, no que se refere à contração de polimerização, as RCBF demonstraram, pequena modificação volumétrica com a fotoativação. Esses resultados decorrem provavelmente por causa da dessemelhança dos elementos compostos e a técnica estabelecida no equipamento de fotoativação. Considerações Finais: As RCBF são recomendadas para a restauração dos elementos dentais posteriores. Contudo, dureza e constrição das reações de polimerização possuem ligação direta com o período e eficácia da emissão irradiadora do fotoativador. Estudos clínicos longitudinais são essenciais para a conjectura do comportamento das RCBF.

PALAVRAS-CHAVE: Reações químicas, Estética, Polimerização, Força Compressiva.

\begin{abstract}
Objective: To evaluate the use of bulk fill composite resins (RCBF) demonstrating their indications and highlight the technique of their procedures with respect to resistances and contractions in photoactivation. Methods: This was a literature review, with a descriptive and informative approach, where the formulation process was through searches for scientific literature in databases, having as descriptors: The importance of the composite resin, the form of polymerization, irradiation power and hardness. Results: In contrast to conventional composite resins, often targeted for addition in $2 \mathrm{~mm}$ increments at the extreme, RCBF expressed better vigor patterns. However, regarding the polymerization shrinkage, the RCBF showed a small volumetric modification with protoactivation. These results are probably due to the dissimilarity of the composite elements and the technique established in the photoactivation equipment. Final Considerations: RCBFs are recommended for restoration of posterior dental elements. However, the hardness and constriction of polymerization reactions are directly linked to the period and effectiveness of the photoactivator irradiation emission. Longitudinal clinical studies are essential for the conjecture of FHR behavior.
\end{abstract}

Key Words: Chemical Reactions, Esthetics, Polymerization, Compressive Strength.

\section{RESUMEN}

Objetivo: Evaluar el uso de resinas compuestas de relleno a granel (RCBF) demostrando sus indicaciones y resaltar la técnica de sus procedimientos con respecto a las resistencias y contracciones en la fotoactivación. Métodos: Esta fue uma revisión de la literatura, con un enfoque descriptivo e informativo, donde el proceso de formulación fue a través de búsquedas de literatura científica en bases de datos, teniendo como

${ }^{1}$ Centro Universitário CESMAC (CESMAC), Maceió-Alagoas. *E-mail: Milton_neto_166@hotmail.com 
descriptores: La importancia de la resina compuesta, la forma de polimerización, poder de irradiación y dureza. Resultados: A diferencia de las resinas compuestas convencionales, a menudo destinadas a la adición en incrementos de $2 \mathrm{~mm}$ em el extremo, RCBF expresó mejores patrones de vigor. Sin embargo, con respecto a la contracción de polimerización, el RCBF mostró una pequena modificación volumétrica con fotoactivación. Estos resultados probablemente se deban a la disimilitud de los elementos compuestos y la técnica establecida en em equipo de fotoactivación. Consideraciones finales: Los RCBF se recomiendan para la restauración de elementos dentales posteriores. Sin embargo, la dureza y la constricción de las reacciones de polimerización están diretamente relacionadas con el período y la emisión de irradiación del fotoactivador. Los estudios clínicos longitudinales son esenciales para la conjetura del comportamiento de la RCBF.

Palabras Clave: Reacciones Químicas, Estética, Polimerizacion, Fuerza Compresiva.

\section{INTRODUÇÃO}

A grande procura por materiais que possuam grande qualidade restauradora nos dentes e que sejam parecidos com as exibidas pelos substratos presentes no elemento dentário é bastante elevada atualmente, sendo sempre levados em consideração na hora da sua escolha: Os aspectos físicos, mecânicos e estéticos (RODRIGUES JUNIOR e COSTA E, 2016).

Sendo as Resinas Compostas (RC) determinadas por poliméricos, onde são na sua complexidade repletos de ligaduras transpassadas e reorganizada por uma disseminação de vidros presentes, além de cristais e porções de cargas orgânicas Anusavice KJ (2005), no decorrer dos tempos foi observado através de estudos que as $\mathrm{RC}$ também são apropriadas para o processo de restaurações de dentes posteriores em permutação com o amálgama e algumas restaurações de prótese que sejam indiretas. Porém os fatores que fazem com quer ocorra um resultado satisfatório e longevidade do procedimento, procede principalmente da aptidão, competência e habilidade técnica do Cirurgião Dentista (CD), da instrução e motivação feita pelo paciente, sendo levado em consideração os hábitos alimentares e a prática da higienização oral, acrescentando a qualidade do material (KAYA MS, et al., 2018).

O processo de polimerização decorrentes das Resinas compostas inicia-se através do mecanismo de adição, onde os radicais livres presentes em sua composição são ativados e degradados através de um processo de fotoativação. O processo do desenvolvimento da modificação de monômeros em cadeias poliméricas lineares ou ramificadas irá depender das características das propriedades e da quantidade da luz que é emitida pelo aparelho fotoativador, onde por sua vez produzirá uma forte tensão no dente-restauração na interface do mesmo, por causa da constrição volumétrica do material composto na RC (ILIE N, et al., 2013).

A fim de certificar a conversão correta da quantidade de RC inserida no preparo restaurador da cavidade dentaria e diminuir as decorrências adversas da contração, ocorreu a determinação da técnica incremental, do qual foi visto resultados mais satisfatórios, dos quais estabeleceu a padronização onde cada incremento de RC deve ter no máximo $2 \mathrm{~mm}$ de densidade (SAKAGUCHI RL, et al., 1993). Contudo, foi observado que esse processo metodológico utilizado, quando são colocados em pratica principalmente em cavidades que possuem uma extremidade e profundidade muito grande exigi muito tempo de trabalho e desgaste dos Cirurgiões Dentistas, além de elevar o risco de contaminações durante o procedimento devido inúmeros fatores correlacionados a contaminação cruzada (JUNG JH e PARK SH, 2017).

Desse modo, para a otimização do atendimento clínico, sem comprometimento e prejudicar a adesão do processo químico estabelecido dente-restauração, sobrechegaram as RC de fixação única ou bulk fill. Segundo os criadores dessa nova RC, as bulk fill possuem indicações para o melhor resultado de preenchimentos cavitários de até 4 a $5 \mathrm{~mm}$ com aplicação única, dado que, em contrapondo as RC convencionais, expressam significada alteração no desconjuntamento da constituição dos compostos e quantidade monomérica (FLURY S, et al., 2014), na ampliação da translucidez (GAROUSHI S, et al., 2016) e na atenuação do composto inorgânico (CHESTERMAN J, et al., 2017).

Entretanto, apesar da perspectiva atual nos referidos estudos de compósitos bulk fill, as literaturas são conflituosas no que se refere o seu desempenho. Por causa da contenção das patentes dos recentes componentes que integram o referido material, não possui meios diretos de avaliá-los de forma particularizada 
e pouco se sabe a respeito da atuação destes sobre a habilidade de conversão e de indução de pressões na interface referente a carga/matriz (CHESTERMAN J, et al., 2017).

Baseado nesses aspectos, o presente estudo tem como principal objetivo realizar uma revisão de literatura sobre a utilização da Resinas Compostas do tipo bulk fill (RCBF) analisando a microdureza e o grau de modificação dos novos compósitos que constituem a RC Bulk Fill, com o intuito de analisar se realmente estes devem ser aplicados clinicamente como material restaurador indireto com grandes propriedades mecânicas.

\section{METODOLOGIA}

Para o desenvolvimento deste trabalho foi realizada uma revisão de literatura, sendo feita entre o período de janeiro de 2019 a setembro de 2019, onde foram analisados artigos publicados em bases de dados eletrônicos LILACS (Literatura Latino Americana e do Caribe em Ciências da Saúde), MEDLINE (Literatura Internacional em Ciência da Saúde) e SCIELO (Scientific Electronic Library Online) e livros que estavam disponíveis nos acervos bibliográficos presentes na biblioteca central do Centro Universitário CESMAC (Centro de Ensino Superior de Maceió).

Foram consultados textos em língua portuguesa, inglesa e espanhola, que se referiram aos protocolos clínicos da utilização das Resinas Compostas do tipo bulk fill (RCBF), tendo como critérios de exclusão os períodos de publicações, os artigos que não estavam na integra, relatos de casos, séries de casos, estudos em animais, ensaios não controlados, monografias e os artigos que não possuíam relevância com a temática, sendo selecionados os textos científicos que apresentavam na integração real objetivo do trabalho, observando a importância da utilização das Resinas Compostas do tipo bulk fill (RCBF) na área odontológica visando o melhor procedimento para a obtenção de resultados satisfatórios e benéficos para o paciente, sendo selecionadas publicações científicas no período entre 2007 e 2019, com ênfase no intervalo entre 2015 e 2019, dispondo dos seguintes descritores: resina composta, dureza, contração, polimerização, tempo de irradiação e potência de irradiação.

\section{RESULTADOS E DISCUSSÃO}

Por causa das suas particularidades no que se refere a estéticas e mecânicas, acrescentado a grande durabilidade clínica, a resina composta é um dos materiais restauradores mais frequentemente escolhido para a restauração direta de dentes tanto anteriores como posteriores (ALKHUDHAIRY FI, 2017).

O surgimento da Resina Composta aconteceu na década de 60, no decorrer dessas últimas décadas ela tem passado por processos evolutivos e consequentemente dispondo de uma área cada vez mais utilizada pelos profissionais da odontologia, devido seus aspectos estéticos e funcionais. Os componentes ativos relacionados ao mecanismo, adesividade e estética está evoluindo constantemente, abrangendo hoje um patamar de elevada importância nas clínica diária. O desempenho evolutivo da $R C$ foi influenciado e aprimorado com a utilização dos fotopolimerizadores (ALKHUDHAIRY FI, 2017).

A utilização da fotopolimerização melhorou o desempenho mecânico, diminuindo por sua vez os problemas relacionados a porosidade e questões de contaminações, porém contribuiu diretamente no aumento de tempo no que se refere aos processos restauradores feitos por esses profissionais. Com a utilização direta dos fotopolimerizadores na RC, os monômeros transformam-se em polímeros estabelecendo uma constrição vertiginosa ocasionando exaustão no interior do material utilizado. Quando não ocorre o controle do estresse no material pode ocorrer vários insucessos (CHESTERMAN J, et al., 2017).

Apesar dos grandes avanços feitos no decorrer das últimas décadas, ainda assim, o processo de contração encontrado na RC devido a polimerização e as limitações quanto a profundidade da cavidade dental na polimerização são características que apresentam desvantagens das RC (ALKHUDHAIRY FI, 2017). Conforme ao nível de intensidade, a atenuação do volume pode acarretar stress na interface adesiva, carregando a constituição de fendas, infiltração marginal, sensibilidade pós-operatória, envolvimento da adesão e grande risco de recorrência de possíveis cáries secundárias (HIRATA R, et al., 2015).

No início do século XXI, com o objetivo da não utilização do amálgama e com a finalidade da busca de melhorias para as características relacionadas a mecânica e os processos biológicos da RC, apareceu 
materiais que se dispuseram a identificar os aspectos do processo de sintetização, visando a disponibilização de flúor e cálcio, estabelecendo dessa forma a possibilidade da utilização de materiais resinosos que conseguiam ser sobreposto em revestimentos de até $5 \mathrm{~mm}$ de espessidão. Neste mesmo tempo foram aplicadas as RC que possuía uma menor viscosidade, denominadas como "flow", buscando serem utilizadas no processo associativo com as resinas proferidas no âmbito da sua compactação (ALSHALI RZ, et al, 2015).

Para poder diminuir as possíveis consequências dos efeitos desconforme da contração, é apontada a técnica incremental, com a adição e fotoativação do acrescimo de RC de $2 \mathrm{~mm}$ (FERRACANE JL, 2011). Porém, quando a cavidade é bastante extensa e profunda, a técnica incremental requer maior quantidade de tempo e consequentemente acarreta maior possibilidade de possíveis contaminações no decorrer dos procedimentos (ALSHALI RZ, et al, 2015).

Inquietos com os fatores limitantes e a carência que eram ligadas às resinas compostas de uso direto, as empresas responsáveis pelos materiais restauradores investiram muito nos últimos anos, pois possuía como objetivo conseguir um melhor material, visando atender os principais parâmetros nos aspectos que englobassem o meio físico, mecânico e estético, onde fossem importantes para a aquisição desses fatores em um único material restaurador (EL-DAMANHOURY H e PLATT J, 2014). Para melhoria da otimização e simplificação dos procedimentos, apareceram as resinas compostas do tipo bulk fill (RCBF), ou RC de preenchimento único. Essa nova linhagem de RC é suscetível de poder ser fotoativada em incremento único de até 4 a $5 \mathrm{~mm}$ (EL-DAMANHOURY H e PLATT J, 2014).

Isso se deu por conta dos pacientes, onde buscavam procedimentos rápidos e que possuíssem resultados satisfatórios, com isso, os fabricantes por sua vez vendo a carência que os materiais possuíam para atender a demanda dos pacientes mais criteriosos buscaram desenvolver uma nova formula que atendessem a esse público alvo, fazendo uma nova versão de resina composta à base de metacrilato, surgindo assim as Bulk Fill. Para os clientes que precisavam de processos restaurativos maiores, essa nova resina apresentou maior comodidade e conforto, já para os Cirurgiões Dentistas ocorreu a redução do tempo de trabalho. Isso foi possibilitado porque as RCBF proporcionavam menor tempo de fotopolimerização e melhor porção de incrementos em uma única aplicação (ALSHALI RZ, et al, 2015).

Elsharkasi $\mathrm{M}$ et al. (2015), em uma análise feita através de estudos com 32 pré-molares observaram a deflexão das cúspides. Foram examinados os preparos cavitários no sentido mésio-ocluso-distal, sendo feita a restauração com a resina Bulk Fill e com a RC. Diante dos resultados dos estudos observou-se que as resina Bulk Fill possuíam pequena deflexão das cúspides quando comparadas as RC, demostrando com isso que essas primeiras, são mais favoráveis para aplicação.

El-Safty S, et al. (2012) ressaltaram que as RC do tipo Bulk fill apresentam particularidades bastante importante para a obtenção de um resultado superior, quando comparadas com as resinas compostas convencionais, sendo elas: boa redução na contração no processo de polimerização, possui grande adaptação na cavidade, boa aptidão para manipulação; aspectos físicos elevados e depressões de polimerização aprimorada. Além de possuir material translúcido que facilita o processo da penetração eletromagnética dos feixes de luzes até a camada mais profunda.

Leprince JG, et al. (2014) fizeram um estudo comparativo com os equipamentos de fotopolimerização, buscando as propriedades adequada para o melhor estabelecimento físico-mecânicos de RC fluidas, resinas Bulk Fill e RC convencionais. Sendo os resultados melhores em resinas convencionais com os respectivos resultados, obtiveram como considerações conclusivas que a as resinas do tipo Bulk Fill não possuíam os princípios de propriedades mecânicas tão favoráveis se não respeitasse o tempo adequado para o processo de polimerização.

Monômeros em polímeros, no decorrer da fotoativação, são analisados por meio da firmeza superficial e da contração ocorrida nos processos de polimerizações, propostas da vigente revisão de literatura (ELDAMANHOURY H e PLATT J, 2014). Com relação ao fator dureza, foram analisadas e avaliadas 5 publicações mais relevantes para o decorrer do estudo (Quadro 1). 
Quadro 1 - Dados obtidos a partir de pesquisas em bases cientificas, distribuídas a partir das referências por autor, ano, objetivo do trabalho e os materiais utilizados.

\begin{tabular}{|c|c|c|c|}
\hline Autores & Anos & Objetivo & Materiais \\
\hline $\begin{array}{l}\text { GONÇALVES } \\
\text { F, CAMPOS } \\
\text { LMP, } \\
\text { RODRIGUES } \\
\text { ECJ, et al. }\end{array}$ & 2018 & $\begin{array}{l}\text { O objetivo do presente estudo foi avaliar } \\
\text { o grau de conversão, encolhimento } \\
\text { volumétrico pósgel e a citotoxicidade de } \\
\text { seis compósitos granulométricos e dois } \\
\text { compósitos convencionais }\end{array}$ & $\begin{array}{l}\text { Oito compósitos comerciais foram testados: } \\
\text { Filtek Z350 XT (Z350) / 3M ESPE; Filtek Z350 } \\
\text { XT fluida (ZF) / 3M ESPE; Aura Bulk Fill (AB) / } \\
\text { SDI; everX Posterior (EP) / GC Europa; } \\
\text { SonicFill (SF) / Kerr; Filtek Bulk Fill Posterior } \\
\text { (FBP) / 3M ESPE; Fluxo de enchimento a } \\
\text { granel Filtek (FBF) / 3M ESPE; Fluxo de } \\
\text { enchimento a granel (VB) da Venus / Heraeus } \\
\text { Kulzer. }\end{array}$ \\
\hline $\begin{array}{ll}\text { TAUBOCK } & \text { T, } \\
\text { JAGER } & \text { F, } \\
\text { ATTIN T. } & \end{array}$ & 2018 & $\begin{array}{l}\text { O objetivo do presente estudo foi } \\
\text { investigar o encolhimento } \\
\text { polimerização, o desenvolvimento } \\
\text { força de contração e o grau de } \\
\text { conversão do monômero em } \\
\text { compósitos bulk fill com base em } \\
\text { dimetacrilato de alta e baixa } \\
\text { viscosidade. }\end{array}$ & $\begin{array}{l}\text { Dois compósitos bulk fill (SDR, base x-tra), dois } \\
\text { compósitos de alta viscosidade (Bulk Ormocer, } \\
\text { SonicFill) e dois materiais compósitos } \\
\text { convencionais (Esthet X flow, Esthet X HD) } \\
\text { foram fotoativados por } 20 \mathrm{~s} \mathrm{em} 1275 \mathrm{~mW} / \mathrm{cm} 2\end{array}$ \\
\hline $\begin{array}{lr}\text { KAYA } & \text { MS. } \\
\text { BAKKAL } & \text { M, } \\
\text { DURMUS } & \text { A et } \\
\text { al. }\end{array}$ & 2018 & $\begin{array}{l}\text { O principal objetivo deste estudo foi } \\
\text { comparar o grau de polimerização da } \\
\text { resina do tipo bulk-fill com três } \\
\text { diferentes } \\
\text { fotopolimerização. }\end{array}$ & $\begin{array}{l}\text { O composto utilizado foi Giomer (Beautifil-Bulk } \\
\text { Restorative), a partir de três unidades } \\
\text { fotopolimerizáveis: polywave (Valo); } \\
\text { monowave (DemiUltra: DU); e um LED de } \\
\text { segunda geração (Optima 10: Opt) }\end{array}$ \\
\hline $\begin{array}{l}\text { CANEPPELE } \\
\text { T, BRESCIANI } \\
\text { E. }\end{array}$ & 2016 & $\begin{array}{l}\text { O objetivo desta revisão é descrever as } \\
\text { características dos materiais } \\
\text { disponíveis no mercado, assim como os } \\
\text { resultados clínicos já descritos na } \\
\text { literatura, na tentativa de informar e dar } \\
\text { suporte para a decisão de emprego ou } \\
\text { não deste tipo de materiais. }\end{array}$ & $\begin{array}{l}\text { Artigos científicos dos anos de } 2014,2015 \text { e } \\
2016 \text {, disponíveis no Pubmed, contendo } \\
\text { trabalhos que comparassem as resinas bulk } \\
\text { com resinas convencionais, para que o } \\
\text { panorama atual pudesse ser traçado. }\end{array}$ \\
\hline $\begin{array}{l}\text { FERREIRA AB } \\
\text { e SILVA NEF }\end{array}$ & 2017 & $\begin{array}{l}\text { Este trabalho tem como objetivo, } \\
\text { mostrar as vantagens do uso clínico das } \\
\text { resinas Bulk Fill em relação às resinas } \\
\text { compostas convencionais. }\end{array}$ & $\begin{array}{l}\text { Foi realizado um levantamento bibliográfico, } \\
\text { com a utilização de artigos científicos, } \\
\text { monografias e publicações que possuem } \\
\text { conteúdos relevantes ao texto deste artigo. } \\
\text { Para isso, foram acessados artigos em } \\
\text { português e inglês, no período entre } 2006 \text { e } \\
2017 \text {, a partir das bases de dados PubMed e } \\
\text { Bireme. }\end{array}$ \\
\hline
\end{tabular}

Fonte: Quadro confeccionado a partir de dados da pesquisa de: Neto JMAS, et al, 2019.

Levando em consideração as suas indicações clínicas como sendo um material de procedimento restaurador para o preenchimento de forma única das cavidades em dentes posteriores, as Resinas Compostas do tipo bulk fill foram criadas e planejadas teoricamente para minimizar possíveis falhas no decorrer da técnica utilizada pelo operador, tendo como objetivo diminuir a quantidade de micro bolhas e consequentemente melhorar a interface adesiva dente-restauração, porém exige um bom conhecimento desse material pelos profissionais Odontológicos (JUNG JH e PARK SH, 2017).

Jung JH e Park SH (2017) ressaltam que é de bastante relevância conseguir alcançar o nível adequado da fotoativação no que se refere as resinas do tipo Bulk Fill, respeitando todas as recomendações e indicações feitas pelos fabricantes, sendo utilizados fotopolimerizadores que possuam ótimas propriedades, e ondas de feixes de luzes com eficácia mínima de $800 \mathrm{~mW} / \mathrm{cm} 2$, estando no parâmetro correto uma potência que exerça $1000 \mathrm{~mW} / \mathrm{cm} 2$, pois tem que ser levado em consideração a perda da luz, já que ela é desvanecida antes mesmo de chegar nas partes mais profundas do processo cavitário. 
No entanto, Taubock T et al. (2018), ressaltou com fundamentos em seu estudo científico, que a RCBF expõe resultados melhores quando contrapõe a RC convencional. Isso em razão de que a RCBF demonstra alterações em seus componentes presentes, disponibilizando maior translucidez. Tal efeito está relacionado diretamente com o maior grau de conversão e, por isso, ocorre o melhoramento nas propriedades físicas. De maneira igual, ao ser comparada a microdureza profunda, as RCBF apresentam resultados mais satisfatórios que a RC convencional, provando que esse compósito novo apresenta uma fotopolimerização homogênea a $5 \mathrm{~mm}$ de profundidade

O nível de alteração das Resinas Compostas são, por resultado, os conceitos de consistência constatado em todas propriedades da RC fotoativada dependendo da qualidade de absorvimento e transmissão da luz (FERREIRA AB e SILVA NEF, 2017). Existem desigualdade relevante nos conceitos de microdureza em todo processo de dilatação da RC fotoativada onde são evoluídas nas RCBF que conseguem um maior nível conversão pertinente (KAYA MS, et al., 2018). Contrário a esses achados, Junior R et al. (2016), analisaram conformidade nos resultados das áreas mais externas nos ângulos e nas bases em RCBF.

Por apresentarem um vazamento superior na cavidade, quando contraposta às $R C$ convencionais, as $R C B F$ certificam um melhor preenchimento de áreas e ângulos difíceis de serem preenchidos. (FERREIRA AB e SILVA NEF, 2017). As contrações decorrentes da polimerização da RC algumas vezes resultam em trincas nas partes mais externas correspondente ao esmalte, acarretando também pressão nas pontas das cúspides e no pós-operatório acontece de imediato sensibilidades. Acrescentado aos resultados da modificação do grau de amplificação térmica linear e à potência oclusal, a constrição até então consegue constituir perda da adesividade e no processo de infiltração marginal, de modo consequente, no pós-operatório ocorre sensibilidade e possíveis caries secundarias (GAROUSHI S, et al., 2016). Taubock T et al. (2018), complementam que as RCBF possuem inferior processo de contração de polimerização quando se é comparada com a RC por consequência da elevada proporção de partículas das cargas.

Inverso a esses estudos, Gonçalves F et al. (2018), verificaram que os estresses ocorridos pelas contrações no processo de condensação são maiores nas RCBF. Uma das possibilidades que podem efetivar esse acontecimento são as alterações dos elementos encontrados nas resinas do tipo Bulk Fill, onde possuem as introduções dos monômeros UDMA (uretano dimetacrilato) e TEGDMA (trietileno glicol dimetacrilato). O UDMA confirma mais elasticidade e menos viscosidade, sendo capaz de ser o cumpridor por baixar a firmeza da resina composta.

\section{CONSIDERAÇÕES FINAIS}

De acordo com a resolução da literatura correlata, foi possível concluir que tanto o processo de contração quanto os valores de dureza desinente da fotoativação das RCBF possuem uma correlação direta com a quantidade do tempo e da potência de irradiação do aparelho fotoativador contribuindo diretamente para o melhor desempenho e agilidade no trabalho dos Cirurgiões Dentistas. Até então, estudos clínicos longitudinais são essenciais para a conjectura do comportamento das Resinas Compostas do tipo bulk fill.

\section{REFERÊNCIAS}

1. ALKHUDHAIRY FI. The effect of curing intensity on mechanical properties of different bulk-fill composite resins. Clin Cosmet Investig Dent, Kingdom of Saudi Arabia, 2017; p.1-6.

2. ALSHALI RZ, et al. Post-irradiation hardness development, chemical softening, and thermal stability of bulk-fill and conventional resin-composites. J Dent, Manchester, v.43, n.2, 2015; p.209-218.

3. ANUSAVICE KJ. Phillips: materiais dentários. 11.ed. Rio de Janeiro: Elsevier, 2005; p. 800.

4. CANEPPELE T, BRESCIANI E. Resinas bulk-fill - O estado da arte. 2016. Revisão de literatura. Departamento de Odontologia Restauradora do Instituto de Ciência e Tecnologia do Campus de São José dos Campos - Unesp, 2016

5. CHESTERMAN J, et al. Bulk-fill resin-based composite restorative materials: a review. British Dental Journal, Manchester, v.222, n.5, 2017; p.337-344.

6. EL-DAMANHOURY H, PLATT J. Polymerization shrinkage stress kinetics and related properties of bulk-fill resin composites. Oper Dent, v.39, n.4, 2014; p.374-82.

7. EL-SAFTY S, et al. Nanomechanical properties of dental resincomposites. Dent Mater 2012; 28:1292-1300 
8. ELSHARKASI M, et al. Cuspal deflection in premolar teeth restored with bulk-fill resinbased composite materials. Tese (Master of Science in Dentistry) - Indiana University School of Dentistry, Indiana, 2015.

9. FERRACANE JL. Resin composite--state of the art. Dent Mater, Portland, v.27, n.1, 2011; p.29-38.

10. FERREIRA AB, SILVA NEF. Utilização das Resinas Compostas Bulk Fill: uma revisão da literatura. 2017. Trabalho de conclusão de curso (Bacharel em Odontologia) - Faculdade Integrada de Pernambuco, Pernambuco, 2017.

11. FLURY S, et al. Influence of increment thickness on microhardness and dentin bond strength of bulk fill resin composites. Dent Mater, Bern, v.30, n.10, 2014; p.1104-12.

12. GAROUSHI S, et al. Influence of increment thickness on light transmission, degree of conversion and micro hardness of bulk fill composites. Odontology, Turku, v.104, n.3, 2016; p.291-7.

13. GONÇALVES F, et al. A comparative study of bulk-fill composites: degree of conversion, post-gel shrinkage and cytotoxicity. Braz Oral Res, São Paulo, 2018; v.32.

14. HIRATA R, et al. Shrinkage assessment of low shrinkage composites using microcomputed tomography. J Biomed Mater Res B Appl Biomater, New York, v.103, n.4, 2015; p.798-806.

15. ILIE N, et al. Influence of various irradiation processes on the mechanical properties and polymerization kinetics of bulk-fill resin-based composites.2013.

16. JUNG JH, PARK SH. Comparison of Polymerization Shrinkage, Physical Properties, and Marginal Adaptation of Flowable and Restorative Bulk Fill ResinBased Composites, Oper Dent, v.42, n.4, 2017; p.375-386.

17. JUNIOR R, COSTA E. Estudo de propriedades de resinas compostas bulk fill. 2015. Dissertação de Mestrado (Odontologia) - USP, São Paulo, 2016.

18. KAYA MS, et al. Structural and mechanical properties of a giomer-based bulk fill restorative in different curing conditions. J Appl Oral Sci, Turkey, 2018.

19. LEPRINCE JG, et al. Physico-mechanical characteristics of commercially available bulkfill composites. J Dent, v. 42 , n. 8, 2014; p. 993-1000.

20. RODRIGUES JUNIOR, COSTA E. Estudo de propriedades de resinas compostas bulk fill. 2015. Dissertação de Mestrado (Odontologia) - USP, São Paulo, 2016.

21. SAKAGUCHI RL, et al. Curing light performance and polymerization of composite restorative materials. J Dent, Minneapolis, v.20, n.3, 1993; p. 183-8.

22. TAUBOCK T, et al. Polymerization shrinkage and shrinkage force kinetics of high- and low-viscosity dimethacrylateand ormocer-based bulk-fill resin composites, Odontology, Zurich, 2018. 